\title{
Longitudinal Impedance of a Prototype Kicker Magnet System
}

\author{
H. J. Tran, M. J. Barnes, G. D. Wait, Y. Yan \\ TRIUMF, 4004 Wesbrook Mall, Vanconver, B.C., Canada V6T 2A3
}

\section{Abstract}

The longitudinal impedance of a kicker magnet system for the proposed KAON factory has been measured from 0.3 to $200 \mathrm{MHz}$. The measurement was done by transforming the kicker magnet under test into a coaxial line in order to measure the transmission parameter $S_{21}$ through the line. The measurement was performed in two frequency ranges. From 0.3 to $50 \mathrm{MHz}$ the magnet was transformed into a $50 \Omega$ coaxial line, and from 45 to $200 \mathrm{MHz}$ into a $180 \Omega$ coaxial line. Resonances in the longitudinal impedance spectrum are due to the electrical resonant modes of the kicker magnet system. The effect on the longitudinal impedance of a speed-up network and a saturating inductor, installed on the input to the kicker magnet to improve its kick performance, was determined. The speedup network can damp some of the resonances whereas the saturating inductor can eliminate the resonances due to the input cable of the kicker magnet. Above $45 \mathrm{MHz}$ where attenuation in the LC cells of the kicker magnet is strong, external components connected to the kicker magnet have negligible influence on the longitudinal impedance. Hence the longitudinal impedance spectrum of the kicker magnet in the 45 to $200 \mathrm{MHz}$ frequency range does not depend on external components such as the speed-up network, the saturating inductor and the input and output cables.

\section{INTRODUCTION}

In the proposed KAON factory a high intensity beam of $100 \mu \mathrm{A}$ is accelerated to $30 \mathrm{GeV}$ step by step in a series of 5 accelerator rings. Kicker magnets will be used to inject and extract the beam. A $1 \mathrm{MHz}$ beam chopper $[1,2]$ will be used to create gaps in the bean during which the field in the kicker magnet must rise or fall from $1 \%$ to $99 \%$ in order to minimize beam losses. The time interval of the gap created is approximately $108 \mathrm{~ns}$ long. The required rise (fall) time of each kicker magnct varies from ring to ring. The fastest rise (fall) time will be $82 \mathrm{~ns}$ [2]. The design of these kicker magnets is based on those of CERN PS Division. Each kicker magnet consists of many LC cells. Fach cell consists of ferrite $\mathrm{C}$-cores sandwiched between high voltage capacitance plates. Hence a characteristic impedance for a kicker magnet can be defined in terms of the inductance and capacitance of its LC cells The injection and extraction kicker magnets in the KAON factory will have a designed characteristic impedance of $25 \Omega$ with the exception of those in the booster ring which will be $16.7 \Omega[2]$.

Longitudinal impedance characterizes the interaction of the beam with accelerator components in the frequency do-

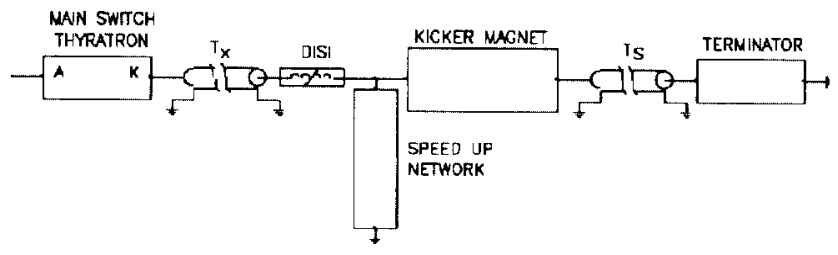

Figure 1: Block diagram of thc kicker magnet system.

main. In terms of equivalent circuit representation, this interaction can be described as mutual coupling between the beam and accelerator components. In order to achieve high beam intensity and energy, collective instabilities driven by longitudinal impedances must be minimized or carefully controlled. Thus all accelerator components which can give a large contribution to the total longitudinal impedance have to be carefully designed and measured before installation. Due to the large number of kicker magnets required in the proposed KAON factory, their contribution to the total longitudinal impedance should be determined.

\section{Kicker Magnet Systems}

A kicker magnet system consists of pulse-forming networks, transmission cables, several kicker magnet modules with speed-up networks and saturating inductors, and resistive or short terminations. Speed-up networks and saturating inductors are components which improve the performance of the kicker magnets $[4,5]$. To avoid reflections in the system, the characteristic impedance of all the components is matched as close as possible. The input cable from the kicker magnet ( $T_{x}$ in Fig. 1) is connected to the main-switch thyatron of the pulse-forming network. Thus when the main-switch thyratron is in the off state, this end of the cable is effectively open circuit (see Fig.1). The output cable $\left(\mathrm{T}_{s}\right.$ in Fig.1) is usually connected to a resistive load. For the extraction kicker magnets in the booster ring, the outputs of the magncts may be shorted [2] and there are no output cables needed.

As part of the K $\Lambda$ ON Factory project definition study a prototype kicker magnet has been designed and built at TRIUMF. PSpice modelling $[3,4]$ has been done to determine optimal values of circuit elements for the speedup networks and saturating inductors. The prototype magnet [2] is wired up for each electrical configuration in which it would operate and then the transmission parameter $S_{21}$ is measured to determine the longitudinal impedance. The effect of the cables, the speed-up network and the displacement-current suppression saturating 
inductor (DISI) on the total longitudinal impedance is determined.

\section{Transformed CoAxial Lines}

We performed longitudinal impedance measurements by transforming the kicker magnet under test into a coaxial line by putting in a central wire in order to measure the transmission parameter $S_{21}$ through the line. The central conductor emulates the charged particle beam. From coaxial transmission theory we can calculate the longitudinal impedance from the transmission parameter $S_{21}$. In order to minimize the presence of unwanted reflections in the line, matching sections are used to maintain as much as possible a constant characteristic impedance throughout the line. We measured the transmission parameter from 0.3 to $200 \mathrm{MHz}$ in two steps. From 0.3 to $50 \mathrm{MHz}$ we transformed the kicker magnet into a $50 \Omega$ coaxial line and from 45 to $200 \mathrm{MHz}$ into a $180 \Omega$ coaxial line. Semi-rigid 50 $\Omega$ test cables were used to connect the transformed coaxial lines to the HP network analyzer. For the $50 \Omega$ line, HP standards and calibration procedures were used to eliminate the systematic errors of the test cables. Similarly for the $180 \Omega$ line, we used the TSD (Through, Short, Delay) calibration method, which can calibrate the measurement assembly from the network analyzer up to the transformed coaxial line [6]. The TSD calibration standards consist of a Through pipe, a Short plate, and a Delay pipe. Error parameters are calculated from the TSD calibration and then used to extract corrected transmission parameter $S_{21}$.

\section{Longltudinal Impedance and Transmission COEFFICIENT}

To obtain the longitudinal impedance, transmission parameter through the transformed coaxial line is measured and compared to that through a section of reference pipe. A series impedance $Z_{l}$, which can be extracted from the transmission parameter, represents the longitudinal impedance of the kicker magnet in the circuit model of the transformed coaxial line. For the reference pipe and the transformed coaxial line, the conductance can be neglected. Thus the resultant propagation constant $\gamma^{\prime}$ of the transformed coaxial line is

$$
\gamma^{\prime}=\sqrt{\left(R+j \omega L+Z_{l}\right)(j \omega C)}
$$

The transmission parameter $S_{21}$ through a line with propagation constant $\gamma^{\prime}$ is $\exp \left(-j \gamma^{\prime} l\right)$ where $l$ is the length of the line. $S_{21}$ can be separated into the real and imaginary parts explicitly to first order as follows [6],

$$
\begin{aligned}
\text { phase }\left[\frac{S_{21}^{\prime}}{S_{21}^{r e f}}\right] \cong-\frac{l X_{l}}{2 Z_{c}} \\
\operatorname{mag} \cdot\left[\frac{S_{21}}{S_{21}^{r e f}}\right] \cong 1-\frac{R_{l} l}{2 Z_{c}}
\end{aligned}
$$

where $Z_{c}=\sqrt{L / C}$ is the characteristic impedance of the transformed line. These two equations can be used to calculate the longitudinal impedance $R_{l}+j X_{l}$ of the magnet under test after transmission measurements are taken for the transformed line and the reference pipe.

\section{Measurements}

To confirm that a calibration is successful, the longitudinal impedance of the brass reference pipe is measured first. The reference pipe should have very low longitudinal resistance since brass is a very good conductor in this frequency range. Typical maximum longitudinal impedance of the brass reference pipe is less than $0.3+\mathrm{j} 0.2 \Omega$ from 0.3 to $50 \mathrm{MHz}$ and less than $1+\mathrm{j} 3 \Omega$ from 45 to $200 \mathrm{MHz}$. The longitudinal reactance for both frequency ranges increases slightly as the frequency increases. This is due to the phase instability of the long test cables caused by a slight variation in length.

The prototype magnet is $345 \mathrm{~mm}$ in length and has ten LC cells with a characteristic impedance of $30 \Omega$. In the proposed KAON factory, kicker magnets will be short circuited or resistively terminated [2]. For a short termination the output of the magnet is electrically shorted and for a resistive termination the output is connected to a matched resistor by a cable.

Below $45 \mathrm{MHz}$ resonances in the impedance spectrum are produced by electrical resonant modes of the kicker magnet system. These resonances are evident in Fig. 2 and correspond to that of a half-wavelength resonator. The maximum longitudinal impedance for the shorted magnet is $32+\mathrm{j} 34 \Omega$ and for the resistively terminated magnet is $26+\mathrm{j} 34 \Omega$. Above $45 \mathrm{MHz}$ the central conductor, which emulates the particle beam, does not couple strongly to other components, which are connected to the magnet, due to the onset of strong attenuation in the LC cells of the magnet. Hence the impedance spectrum from 45 to 200 $\mathrm{MHz}$ does not depend much on the termination of the magnet nor on other components of the kicker magnet system.

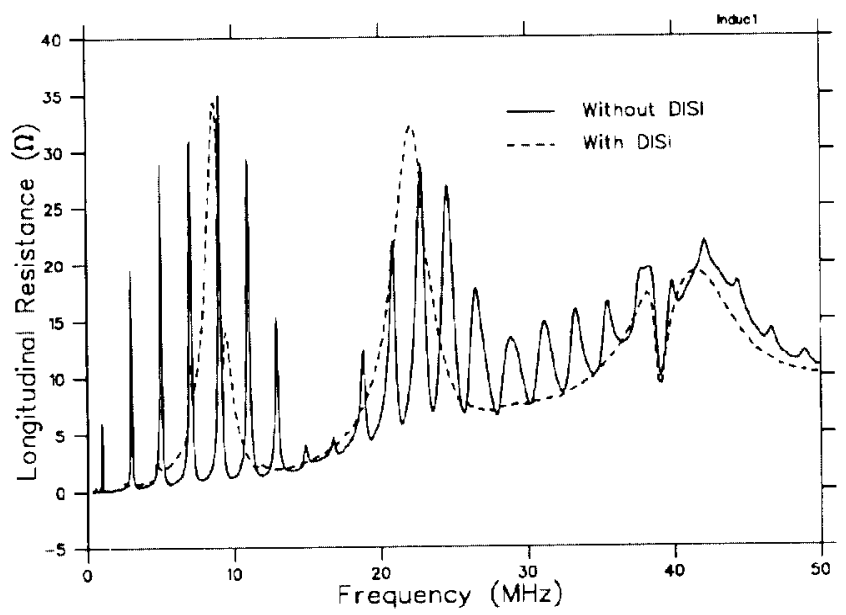

Figure 2: Longitudinal impedance of shorted kicker magnet with and without DISI (No speed-up network, $T_{x}=192 \mathrm{~ns}$ ). 
The maximum impedance is $30+\mathrm{j} 125 \Omega$ in the 45 to 200 $\mathrm{MHz}$ range for both short and resistively terminated kicker magnets. In the 45 to $200 \mathrm{MHz}$ range, the impedance spectrum exhibits small resonances and a rising slope.

It has bcen proposed that a saturating inductor be connected in series between the input of the magnet and the cable $\mathrm{T}_{x}$ (Fig. 1) to absorb small displacement current pulses before the main power pulse so as to improve the rise time of the magnet [1]. For small magnitudes of current the saturating inductor has a high impedance, hence effectively terminating the input cable as an open circuit. Resonances due to the input cable are eliminated when the saturating inductor is connected (Fig.2). The resulting impedance spectrum has the same features as that of a magnet with its input open circuit. Fig.2 shows the impedance spectrum of a shorted magnet with and without the saturating inductor (DISI).

The effect of various speed-up networks with different values of capacitance and resistance is shown in Fig. 4. A speed-up network is connected on the input of the magnet to improve its performance [3,4]. A capacitor and a resistor in series make up a speed-up network. Besides shifting the resonances slightly, the resistor of the network can damp some of the resonances. Hence it is beneficial to connect the speed-up network between the kicker magnet and the saturating inductor. In the proposed KAON factory, a gap may be present between the kicker magnet, which is in a vacuum tank, and the beam pipe which is connected to the tank. Such a gap can contributes additional low and high $\mathrm{Q}$ resonances with large values compared to the longitudinal impedance of the magnet system (see Fig.4).

\section{Conclusion}

We have determined the longitudinal impedance of a kicker magnet system with short and resistive terminations and the effect on the longitudinal impedance of saturating inductors and speed-up networks, which are installed to improve the kick performance. When a saturating induc-

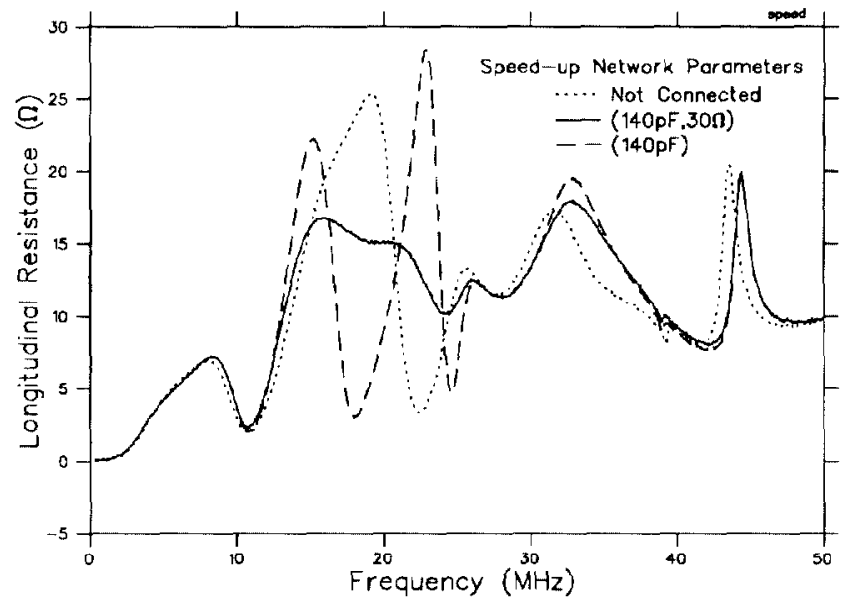

Figure 3: Longitudinal resistance of kicker magnet with speed-up networks (no DISI, $\mathrm{T}_{x}=30 \mathrm{~ns}$ ). tor (DISI) is connected between the input of the magnet and the input cable, the longitudinal impedance spectrum does not contain the resonances which are otherwise produced by the input cable. The resistor of the speed-up network, which is connccted to the input of the magnet, has a beneficial damping effect on some of the resonances. However, in the frequency range from 15 to $200 \mathrm{MHz}$, the longitudinal impedance spectrum does not depend much on the termination of the magnet nor on other components connected to it due to the onset of strong attenuation in its LC cells. Air gap between the kicker magnet and the beam pipe can contribute very large resonances to the longitudinal impedance spectrum of the kicker magnet system

\section{REFERENCES}

[1] G. D. Wait, M. J. Barnes, D. Bishop, G. Waters, "Interleaved Wide and Narrow Pulses for the KAON Factory $1 \mathrm{MHz}$ Beam Chopper" Proceedings of this Conference.

[2] M. J. Barnes, G. D. Wait, "Kickers for the Kaon Factory" Proceedings of $\mathrm{XV}^{t / 2}$ International Conference of High Energy Accelerators (HEACC'92), Hamburg, July 1992.

[3] M. J. Barnes, G. D. Wait. "Optimization of Speed-Up Network Component Values for the $30 \Omega$ Resistively Terminated Prototype Kicker Magnet" Proceedings of this Conference.

[4] M. J. Barnes, G. D. Wait, "Improving the Performance of Kicker Magnet Systems"Proceedings of XV ${ }^{t h}$ International Conference of High Energy Accelerators (HEACC'92), Hamburg, July 1992.

[5] M. J. Barnes and G. D. Wait, "A Mathematical Model of a Three-Gap Thyratron Simulating Turn-on " to be presented at the Ninth IEEE Pulsed Power Conference, June 1993. Albequrque

[6] L.S. Walling et al, "Transmission Line Impedance Measurements for an Advanced Hadron Facility" LANL 1988.

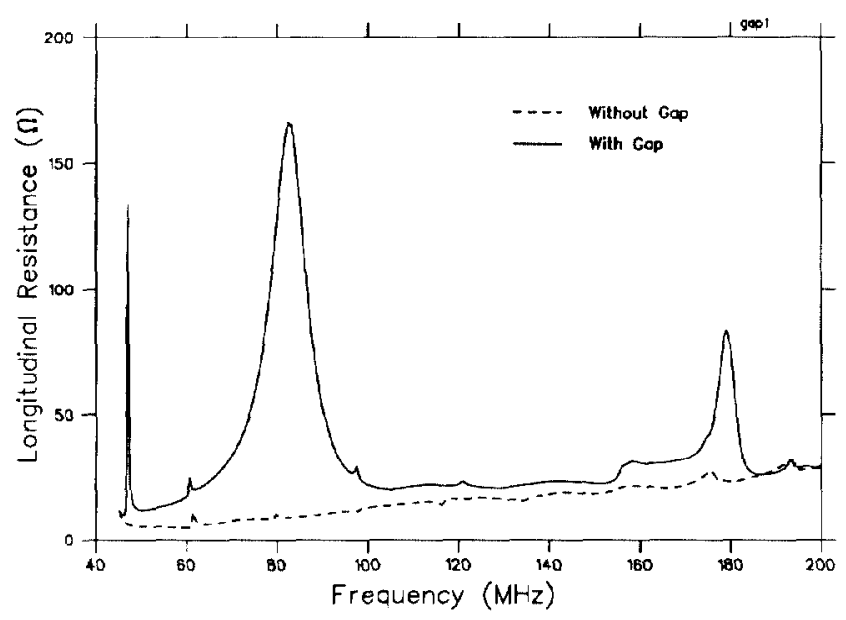

Figure 4: Resonances due to gap. 